\title{
S1PR1 mediates anti-apoptotic/pro-proliferative processes in human acute myeloid leukemia cells
}

\author{
XIAO-QIAN XU ${ }^{1 *}$, CHONG-MEI HUANG $^{1 *}$, YI-FAN ZHANG ${ }^{2}$, LI CHEN $^{1}$, HUI CHENG $^{1}$ and JIAN-MIN WANG ${ }^{1}$ \\ ${ }^{1}$ Department of Hematology, Changhai Hospital, Second Military Medical University, Shanghai 200433; \\ ${ }^{2}$ Department of Nuclear Medicine, Ruijin Hospital, School of Medicine, Shanghai Jiao Tong University, \\ Shanghai 200025, P.R. China
}

Received August 3, 2015; Accepted July 15, 2016

DOI: $10.3892 / \mathrm{mmr} .2016 .5629$

\begin{abstract}
Sphingosine-1-phosphate receptor 1 (S1PR1), the G protein-coupled receptor 1 of sphingosine 1-phosphate (S1P), is crucial in the progression of various types of solid tumor through the positive regulation of anti-apoptotic/pro-survival responses by S1P-S1PR1 signaling. However, the role of S1PR1 in the progression of human acute myeloid leukemia (AML) remains to be fully elucidated. In the present study, the correlation between the expression level of S1PR1 and the growth and apoptosis of human AML cells was investigated. Using overexpression and RNA interference knockdown assays, it was shown that the expression of S1PR1 in several human myeloid leukemia cell lines contributed to the suppression of cell apoptosis and promotion of cell proliferation. S1PR1 inhibited the expression level of B cell lymphoma-2-associated X protein, a mitochondrion-associated apoptosis promoter, and prevented caspase-3 cleavage, indicating that S1PR1 interfered with the mitochondrion-associated apoptotic processes in AML cells. In addition, the present study demonstrated that S1PR1 suppressed the generation of reactive oxygen species (ROS) in AML cells, and that the apoptosis mediated by the downregulation of S1PR1 was partially reversed by treatment with $\mathrm{N}$-acetyl-L-cysteine, a ROS scavenging agent, suggesting that the S1PR1-induced resistance of cell apoptosis resulted, at least partially, from the suppression of ROS generation. Additionally, S1PR1 was able to interfere with the signaling of c-Jun N-terminal kinase, which may have also contribute dto the suppression of cell apoptosis. By contrast, extracellular signal-regulated kinase (ERK) signaling appeared to be positively modulated by the S1PR1-induced enhancement
\end{abstract}

Correspondence to: Dr Jian-Min Wang, Department of Hematology, Changhai Hospital, Second Military Medical University, 168 Changhai Road, Shanghai 200433, P.R. China E-mail: ellenxxq@qq.com

*Contributed equally

Key words: sphingosine 1-phosphate, anti-apoptotic, pro-proliferative, $\mathrm{G}$ protein-coupled receptor of mitogen-activated protein kinase (MAPK) kinase 1 (MKK1) activation, suggesting that S1PR1 may promote cell survival and proliferation through enhancing the activation of MKK1-ERK signaling. In conclusion, S1PR1 likely serves as an anti-apoptotic/pro-proliferative protein in AML cells through the inhibition of mitochondrion-associated apoptosis and ROS generation, and via the regulation of multiple MAPK signaling cascades.

\section{Introduction}

Sphingosine-1-phosphate receptor 1 (S1PR1) belongs to the $\mathrm{G}$ protein-coupled receptor family for sphingosine 1-phosphate (S1P), a biologically active lipid. S1P-S1PR1 signaling is vital in a variety of developmental processes, including cell growth, survival and migration (1). However, S1PR1 signaling also promotes tumor progression and tumor angiogenesis $(2,3)$. In myeloid cells derived from tumors, including activated B cell-like diffuse large B-cell lymphoma, the expression of S1PR1 is elevated, compared with normal splenic myeloid cells, and this upregulation is correlated with the persistent activation of signal transducer and activator of transcription 3 (STAT3) (2,3). S1PR1-STAT3 signaling is also crucial for myeloid cell colonization at future metastatic sites (4). Previous data has suggested a correlation between reduced S1PR1 and reduced survival rates of patients with glioblastoma (5), and the high level of S1PR1 in T-lymphoblastic lymphoma (T-LBL) is a key factor in the inhibition of progression of T-LBL to acute T-lymphoblastic leukemia (6). Studies based on certain S1PR1 inhibitors have also suggested that the degradation of S1PR1 can arrest cell growth in various cancer cell lines (6-8).

Cell growth and apoptosis involve several signaling pathways. The direct executors of apoptosis are intracellular cysteine proteases, termed caspases (9). Caspases are originally translated as inactive zymogens, and subsequently activated by 'mitochondria' or the 'death receptor' pathway. Of note, the release of cytochrome $c$ from mitochondria, which triggers caspase activation, appears to be predominantly mediated by the direct or indirect actions of reactive oxygen species (ROS) (10). The response of tumor cells to cell death stimuli is a consequence of cellular redox status, and stimuli, which induce generation of intracellular ROS facilitate the execution of cell death (11). ROS, including hydroxyl, superoxide, 
nitroxyl radicals, hydrogen peroxide, organic hydroperoxides and hypochlorous acid, can destroy DNA and proteins, and activate cell death $(11,12)$. ROS generation has been shown to be associated with the activation of c-Jun N-terminal kinase (JNK)/stress-activated protein kinase by oxidative inactivation of endogenous JNK inhibitors, including JNK phosphatase receptor-mediated cell death (13-15). JNK and other members of the mitogen-activated protein kinase (MAPK) family, including extracellular signal-regulated kinase (ERK)1/2 and p38 MAPK, are involved in cell proliferation and apoptosis through the activation of specific transcription factors.

With the lowest survival rate among the four major types of leukemia, acute myeloid leukemia (AML) is a significant threat to human health (16). Clinically, AML is characterized as the abnormal differentiation and proliferation of immature blast cells in the bone marrow, and is known to be the most common form of acute leukemia among adults (16). However, the pathogenesis of the disease remains to be elucidated.

Although several studies have suggested that S1PR1 is a notable factor for solid tumors, the role of S1PR1 in AML cell growth remains to be fully elucidated (17). In the present study, the effects of S1PR1 in modulating the apoptosis and proliferation of AML cells were investigated. It was found that S1PR1 signaling mediated anti-apoptotic/pro-proliferative processes in the AML cells through the inhibition of mitochondrion-associated apoptosis and ROS generation, and via the regulation of multiple MAPK signaling cascades.

\section{Materials and methods}

Cell culture and reagents. The human promyelocytic leukemia cell lines, HL60, U937 and K562, were purchased from American Type Culture Collection (Manassas, CA, USA) and propagated in RPMI 1640 (Gibco; Thermo Fisher Scientific, Inc., Waltham, MA, USA) supplemented with $10 \%$ fetal bovine serum (FBS; Gibco; Thermo Fisher Scientific, Inc.) in a $37^{\circ} \mathrm{C}$ incubator with $5 \% \mathrm{CO}_{2}$. The 2-7-dichlorofluorescin diacetate (DCFH-DA) and N-acetyl-L-cysteine (NAC) were purchased from Sigma-Aldrich (MO, St. Louis, MA, USA) and dissolved in saline solution at $\mathrm{pH}$ 7.0.

Overexpression and knockdown of S1PR1. cDNA was synthesized from RNA using a cDNA synthesis kit (Thermo Fisher Scientific, Inc.). Prior to reverse transcription, RNA was treated with DNase (Invitrogen; Thermo Fisher Scientific, Inc.). The coding sequence of S1PR1 was obtained by polymerase chain reaction (PCR), using cDNA generated from the RNA of HL60 as a template, with the following primers: S1PR1, forward 5'-ATGGATGACCTAATCGCCAGA CAC-3' and reverse 5'TTAAGAAGAAGAGGTGATATT-3'. The coding sequence was annealed and digested using AgeI and EcoRI, and then was ligated into pCDNA3 (Invitrogen; Thermo Fisher Scientific, Inc.). The recombinant plasmid was verified by direct sequencing and used to induce the overexpression of S1PR1 in the HL60 cell lines. The siRNA sequence target for S1PR1 was 5'-ATGATCGATCATCTA TAGCAA-3' (18), and a non-silencing small interfering (si) RNA was used as a control (Life Technologies, Grand Island, NY, USA). For transfection and the analysis of expression levels, a total of $1 \times 10^{6} \mathrm{HL} 60$ cells were suspended in $1 \mathrm{ml}$
OPTI-MEM and subsequently transfected with 100 pM siRNA or $4 \mu \mathrm{g}$ pCDNA3-S1PR1 using Lipofectamine 2000 (Invitrogen; Thermo Fisher Scientific, Inc.). After 48 h, the expression of S1PR1 was analyzed by quantitative (q)PCR using a SYBR Green PCR kit (Toyobo, Tokyo, Japan) with the 7300 Real-Time PCR system (Applied Biosystems, Darmstadt, Germany). Expression levels were normalized to GAPDH and measured in triplicate. The following primers were used: S1PR1, forward 5'-CCTCGGTGGTGTTCATTC-3' and reverse 5'-GCAGGTTAGCTGTGTAGG-3'; GAPDH, forward 5'-CACCCACTCCTCCACCTTTG-3' and reverse 5'-CCA CCACCCTGTTGCTGTAG-3'. The PCR cycling conditions were as follows: $95^{\circ} \mathrm{C}$ for $10 \mathrm{~min}$, followed by 40 cycles at $95^{\circ} \mathrm{C}$ for $15 \mathrm{sec}$ and $60^{\circ} \mathrm{C}$ for $45 \mathrm{sec}$, and a final extension step of $95^{\circ} \mathrm{C}$ for $15 \mathrm{sec}, 60^{\circ} \mathrm{C}$ for $1 \mathrm{~min}, 95^{\circ} \mathrm{C}$ for $15 \mathrm{sec}$ and $60^{\circ} \mathrm{C}$ for $15 \mathrm{sec}$. The gene expression was calculated using the $2^{-\Delta \Delta \mathrm{Cq}}$ method (19).

Western blot analysis. Whole cell protein extracts were isolated using RIPA buffer containing $50 \mathrm{mM}$ Tris- $\mathrm{HCl}$, (pH 8.0), 150 mM NaCl, 1\% Nonidet P-40, 0.1\% SDS, 2 mM phenylmethylsulfonyl fluoride, phosphatase and protease inhibitor cocktail (CalbioChem, San Diego, CA, USA) at $4^{\circ} \mathrm{C}$ for $20 \mathrm{~min}$, followed by centrifugation at $12,000 \mathrm{~g}$ for $1 \mathrm{~min}$ at $25^{\circ} \mathrm{C}$. The supernatants were then subjected to SDS-PAGE (Bio-Rad Laboratories, Inc., Richmond, CA, USA). The protein samples were consequently transferred onto PVDF membrane (0.22 $\mu \mathrm{m}$; EMD Millipore, Billerica, MA, USA) using a wet transfer device (Bio-Rad Laboratories, Inc.), followed by blotting with primary antibodies specific for B cell lymphoma-2 (Bcl-2)-associated X protein (Bax; cat. no, Sc-493), Bcl2 (cat. no. Sc-492; Santa Cruz Biotechnology, Inc., Santa Cruz, CA, USA), Caspase 3 (cat. no. Ab32351), phosphorylated (p)-c-Jun (cat. no Ab32385), Jun (cat. no. Ab32137), MKK1 (cat. no. Ab139343), p-MKK1 (cat. no. Ab3338) from Abcam, and p38 (8690), p-p38 (cat. no. 4631), ERK (cat. no. 9102), p-ERK (cat. no. 5726), JNK (cat. no. 9258), p-JNK (cat. no. 5291), GAPDH (cat. no. 5174) from Cell Signaling Technology, Inc. (Beverly, MA, USA) for $2 \mathrm{~h}$ at $25^{\circ} \mathrm{C}$, prior to incubation with secondary antibodies for $1 \mathrm{~h}$ at $37^{\circ} \mathrm{C}$, and were subsequently washed three times with Tris-buffered saline with $20 \%$ Tween-20 (Amresco, LLC, Solon, OH, USA). The secondary antibodies used were horseradish peroxidase-conjugated goat anti-rabbit IgG (cat. no. A0216; Beyotime Institute of Biotechnology, Shainghai, China) or horseradish peroxidase-conjugated goat anti-mouse IgG (cat. no. A0208; Beyotime Institute of Biotechnology). The bands on the membrane were developed using an ECL kit (EMD Millipore) and visualized by exposure to a UVP bio-imaging system (UVP, Inc., Upland, CA, USA).

Cell apoptosis and viability assays. Cell apoptosis was detected using a Fluorescein isothiocyanate (FITC) Annexin V Apoptosis Detection kit with propidium iodide (PI; BioLegend, Inc., San Diego, CA, USA). Briefly, the cell samples were washed twice with ice-cold Annexin V binding buffer, and then stained with Annexin V-FITC and PI for $15 \mathrm{~min}$ at room temperature, followed by the addition of another $400 \mu \mathrm{l}$ Annexin $\mathrm{V}$ binding buffer and analysis using flow cytometry (Accuri C6; BD Biosciences, San Diego, CA, 
USA). Early stage apoptotic cells were stained with FITC Annexin V, but not PI, whereas late stage apoptotic cells and necrotic cells stained positively for FITC Annexin V and PI. Cell viability was assessed using a Cell Counting Kit-8 (CCK-8; Dojindo Laboratories, Kumamoto, Japan), according to the manufacture's protocol. Briefly, $2.5 \times 10^{3}$ target cells were seeded in triplicate into a 96 well plate, following which $10 \mu \mathrm{l}$ of 2-(2-methoxy-4-nitrophenyl)-3-(4-nitrophenyl)5-(2,4-disulfophenyl)-2H-tetrazolium, monosodium salt (WST-8) solution was added to each well and incubated for $4 \mathrm{~h}$ in the incubator. The viable cell-mediated formation of WST-8 formazan was measured at an absorbance of $450 \mathrm{~nm}$ using a microplate reader.

Analysis of intracellular ROS levels. To assess the involvement of S1PR1 in the reduction of ROS, the HL60 cells were transfected with S1PR1 constructs, with or without pre-treatment with $100 \mu \mathrm{M}$ NAC for $2 \mathrm{~h}$. After $48 \mathrm{~h}$, the cell samples were incubated with $5 \mathrm{M}$ of the redox-sensitive dye, DCF-DA, for $30 \mathrm{~min}$ at $37^{\circ} \mathrm{C}$. The ROS-mediated oxidation of the fluorescent compound, DCF, was measured by excitation at $488 \mathrm{~nm}$ and an emission wavelength of $525 \mathrm{~nm}$ using a flow cytometer (BD Biosciences).

Statistical analysis. Data are presented as the mean \pm standard deviation. Statistical analysis was performed by two-way analysis of variance followed by Tukey's test. Statistical analyses were performed using GraphPad Prism software, version 5 (GraphPad Software, Inc., La Jolla, CA, USA). P<0.05 was considered to indicate a statistically significant difference.

\section{Results}

Modulation of the expression of SIPRI by transient transfection with plasmids. To elucidate the effects of S1PR1 in myeloid leukemia cell proliferation and apoptosis, the present study first constructed a mammalian cell expression plasmid for the overexpression of S1PR1 and an siRNA plasmid for knockdown of the expression of S1PR1. As shown in the qPCR assay, the mRNA level of S1PR1 was increased markedly in the HL60 cells transfected with the S1PR1 expression plasmid, whereas transfection with the siRNA plasmid led to a significant reduction in the endogenous expression of S1PR1 (Fig. 1A; P<0.001).

S1PR1 inhibits the apoptosis of human myeloid leukemia cells and promotes their proliferation. The present study examined the effects of the expression of S1PR1 on the proliferation of human myeloid leukemia cells using a CCK-8 kit. The U937 cells were transfected with the siRNA plasmid, the S1RP1 expression plasmid, or the corresponding control plasmids. Cell proliferation was measured at 0,24 and $48 \mathrm{~h}$ post-transfection using a CCK-8 assay. As shown in Fig. 1B, knockdown of the expression of S1PR1 by transfection with the siRNA plasmid significantly inhibited U937 cell proliferation, and the overexpression of S1PR1 appeared to result in a marginal enhancement of cell proliferation. To investigate whether S1PR1 contributes to the inhibition of cell apoptosis, the effect of S1PR1 on cell apoptosis was examined by Annexin-PI staining and flow cytometry. As shown in Fig. 1C, knockdown of the expression of S1PR1 by transfection with the siRNA plasmid markedly induced the apoptosis of leukemia cells, whereas cell apoptosis appeared to be inhibited by the overexpression of S1PR1, suggesting that S1PR1 signaling likely led to the suppression of apoptosis and the promotion of proliferation in the human leukemia cells.

SIPRI confers resistance to mitochondrion-associated apoptosis in myeloid leukemia cells. The apoptotic process in cells is strictly controlled by the expression of anti- and pro-apoptotic proteins. For example, the Bcl-2 family contributes to the regulation of cell apoptosis by functioning as promoters, including Bax, or inhibitors, including Bcl-2 of mitochondrion-associated apoptosis. To further elucidate the molecular mechanism by which S1PR1 suppresses the apoptosis of human myeloid leukemia cells, the present study assessed the protein expression levels of pro-apoptotic Bax and anti-apoptotic Bcl-2, in the context of the overexpression or downregulation of S1PR1. As shown in Fig. 2, the overexpression of S1PR1 resulted in a significant increase in the expression of Bax, thus reducing the ratio of $\mathrm{Bax} / \mathrm{Bcl}-2$. This suggested that S1PR 1 affected cell apoptosis by regulating the expression of mitochondrion-associated anti- and pro-apoptotic proteins. In addition, the effects of the expression of S1PR1 on the activation of caspase-3 were examined. There was an increase in the level of cleaved caspase-3 (17 kD) in the cells with downregulated levels of S1PR1, whereas the overexpression of S1PR1 led to the inhibition of caspase-3 cleavage (Fig. 2). Taken together, these data demonstrated that S1PR1 interfered with myeloid leukemia cell apoptosis via the suppression of mitochondrion-associated apoptotic processes by regulating the expression of Bax and activation of caspase-3.

SIPR1 suppresses the generation of ROS. Several previous studies have shown that ROS is important in stress-induced apoptosis. During cellular stress, ROS can act directly on the apoptotic machinery, by accelerating depolarization and causing dysfunction of mitochondria (20), which in turn, functions as a major source of ROS in the course of apoptosis (21). To investigate whether ROS is involved in the regulation of S1PR1-mediated myeloid leukemia cell apoptosis, the present study compared the levels of ROS in cells with S1PR1 overexpression or knockdown with the control cells. As shown in the results of the flow cytometric analysis (Fig. 3), the downregulation of S1PR1 led to a marked increase in the intracellular levels of ROS. By contrast, the generation of ROS in cells overexpressing S1PR1 was significantly suppressed. To further confirm the involvement of ROS in the S1PR1-mediated regulation of cell apoptosis, NAC, a ROS scavenging agent was used in flow cytometric analysis of S1PR1-mediated regulation of cell apoptosis. As expected, cell apoptosis induced by the downregulation of S1PR1 was partially reversed in cells treated with NAC, further indicating the direct implication of ROS in the S1PR1-mediated resistance of cell apoptosis. These results suggested that S1PR1 confers resistance to cell apoptosis likely by suppressing the generation or accelerating the elimination of ROS.

S1PR1 inhibits JNK-c-Jun signaling. Apart from the direct action of ROS on mitochondrial apoptotic machinery, ROS 
A

C
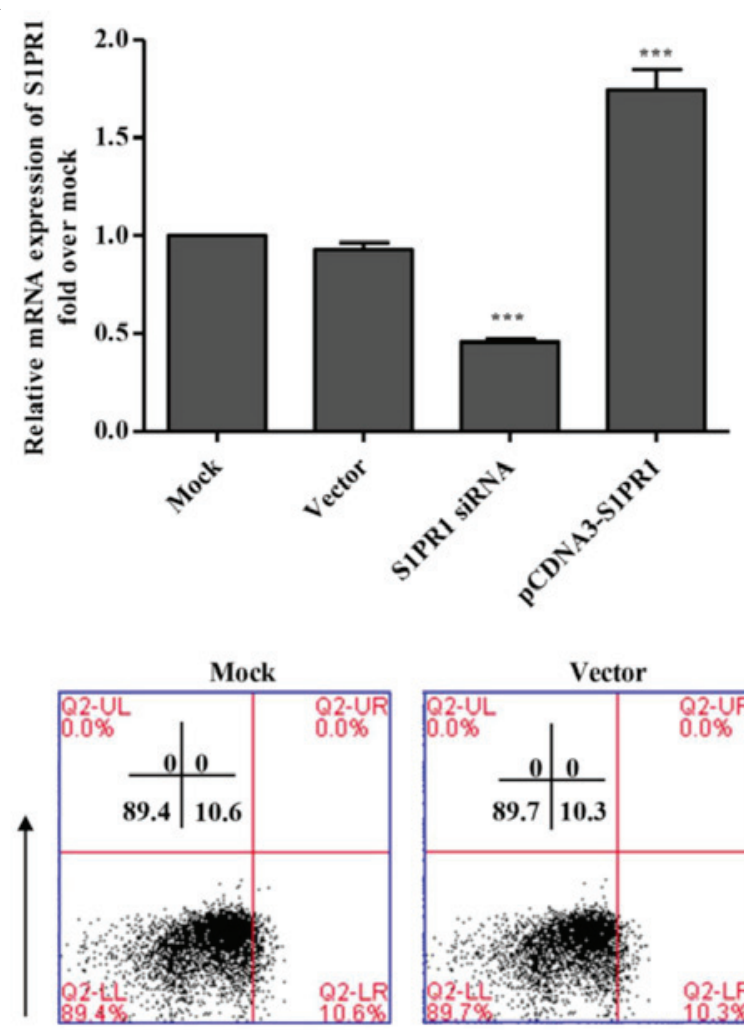

$\overline{2}$

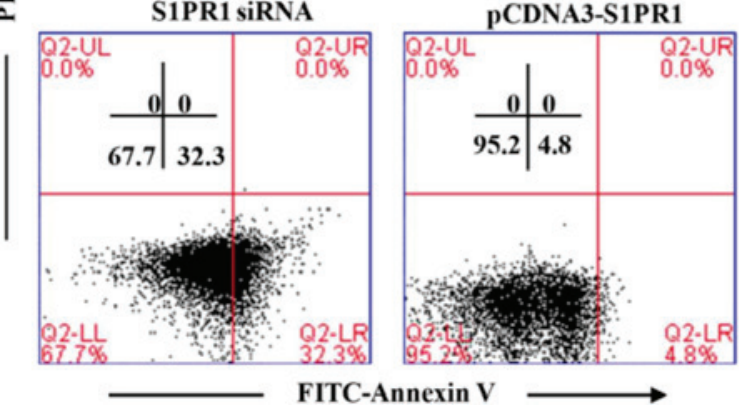

B
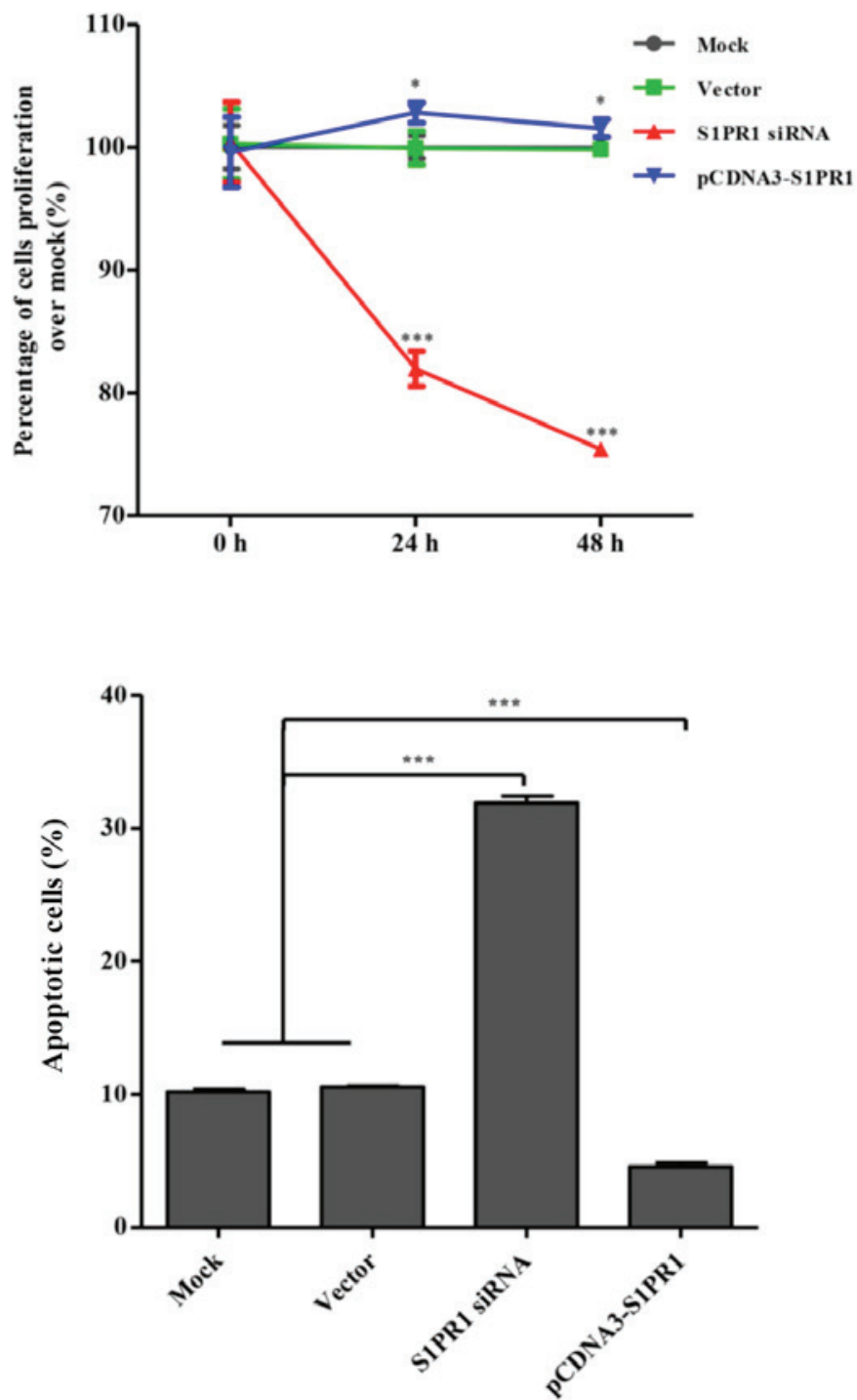

Figure 1. Effects of the expression of S1PR1 on the proliferation and apoptosis of AML cells. (A) Modulation of the expression of S1PR1 by transient transfection with plasmids. HL60 cells were transfected with the indicated plasmids. At 48 h post-transfection, total RNA was isolated from the cell samples and the mRNA levels of S1PR1 were analyzed using quantitative polymerase chain reaction analysis. Data are presented as the mean \pm standard deviation ( $=3$ ). (B) Effects of the expression of S1PR1 on the proliferation of human AML cells. U937 cells were transfected with the indicated plasmids. Cell proliferation was measured at the indicated times post-transfection using a CCK-8 assay. Data are presented as the mean \pm standard deviation ( $\mathrm{n}=3$ ). (C) Effects of the expression of S1PR1 on apoptosis of human AML cells. U937 cells were transfected with S1PR1 siRNA or overexpression plasmids, or control plasmids. After $48 \mathrm{~h}$, cell apoptosis was analyzed with Annexin-PI staining using flow cytometry. A representative assay is shown, with data presented as the mean \pm standard deviation $(\mathrm{n}=3)$. ${ }^{*} \mathrm{P}<0.05$ and ${ }^{*}{ }^{*} \mathrm{P}<0.001$, compared with mock and vector. S1PR1, sphingosine-1-phosphate receptor 1; AML, acute myeloid leukemia; siRNA, small interfering RNA; FITC, fluorescein isothiocyanate; PI, propidium iodide.

can also promote apoptosis by triggering the pro-apoptotic signaling pathway mediated by JNK and p38 MAPK. As ROS is involved in the regulation of cell apoptosis by S1PR1, it was hypothesized that the regulation may also involve ROS-induced JNK or/and P38 MAPK signaling. To confirm this hypothesis, the activation of JNK and P38 were examined by western blot analysis using antibodies against corresponding phosphorylated proteins. The phosphorylation of $\mathrm{p} 38$ was not affected by either the overexpression or knockdown of S1PR1. However, JNK activation was not changed in S1PR1-overexpressing cells, and the knockdown of S1PR1 appeared to induce a significant increase in JNK phosphorylation, compared with the controls (Fig. 4A), suggesting that the downregulated expression of S1PR1 contributed to the activation of JNK. Similar results were obtained from the investigation of the level of c-Jun phosphorylation downstream of the JNK signaling. These findings indicated that S1PR1 specifically interfered with JNK signaling and likely inhibited cell apoptosis.

S1PR1 positively modulates MKK1-ERK signaling, likely resulting in the promotion of cell proliferation. In parallel with JNK and P38, ERK is a member of the MAPK superfamily. However, JNK and p38 MAPK are preferentially activated by proinflammatory cytokines and oxidative stress, resulting in cell apoptosis, whereas ERK1/2 are activated by mitogens and growth factors, leading to cell growth and survival. Thus, the present study evaluated the activation of ERK. The overexpression of S1PR1 increased the phos- 
A

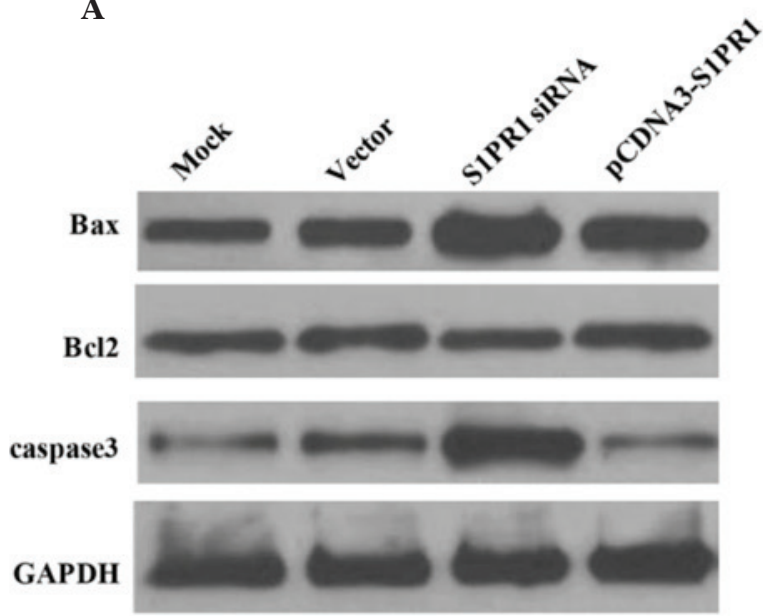

B

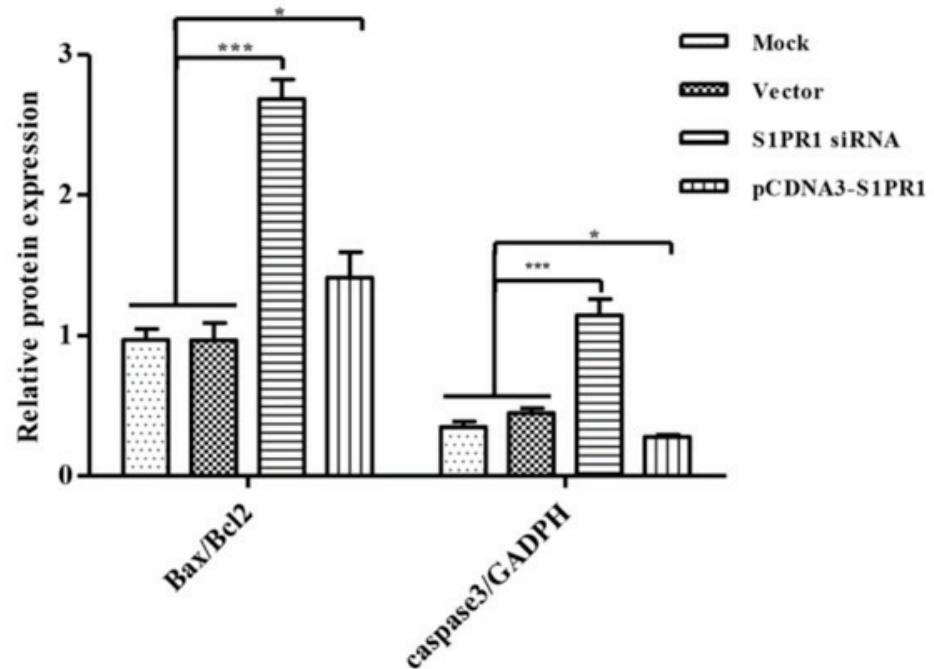

Figure 2. S1PR1 confers resistance to mitochondrion-associated apoptosis on acute myeloid leukemia cells. Cells were transfected with the indicated plasmids and lysed for western blot analysis $24 \mathrm{~h}$ post-transfection using antibodies against the indicated proteins. GAPDH was detected as a control for sample loading. (A) A representative assay is shown. (B) Intensities of the Bax and Bcl2 bands were analyzed and the ratios of Bax/Bcl2 are shown. The protein expression level of caspase 3 was also analyzed. Data are presented as the mean \pm standard deviation. $\mathrm{P}<0.05,{ }^{* * * *} \mathrm{P}<0.001$. S1PR1, sphingosine-1-phosphate receptor 1; Bcl2, B cell lymphoma-2; Bax, Bcl2-associated X protein; siRNA, small interfering RNA.

A
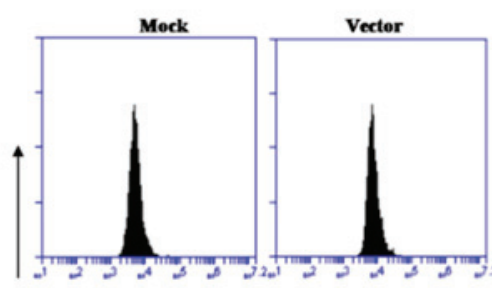

套

SIPR1 siRNA

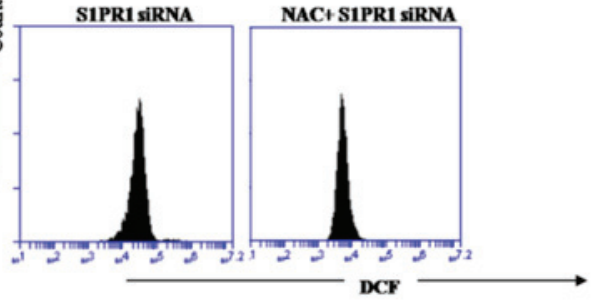

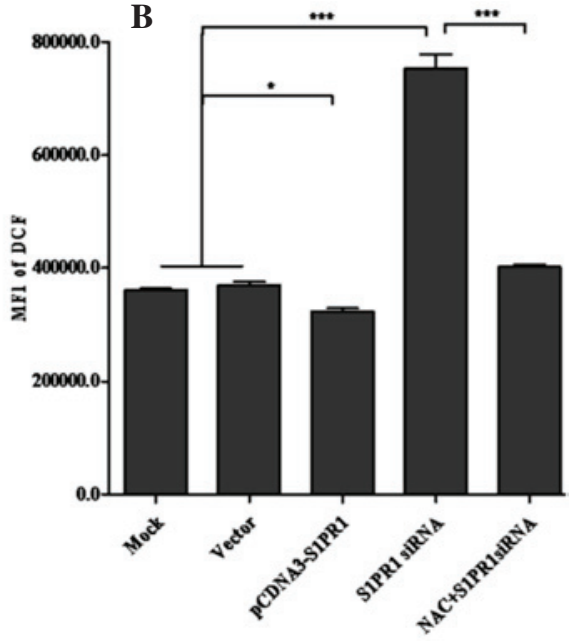

Figure 3. Effects of the expression of S1PR1 on the generation of ROS in AML cells. The cells were transfected with the indicated plasmids, and the levels of intracellular ROS were analyzed using flow cytometry. (A) A representative assay is shown. (B) Mean fluorescence intensity (MFI) of DCF values from three independent experiments are presented as the mean \pm standard deviation. ${ }^{*} \mathrm{P}<0.05,{ }^{* * * *} \mathrm{P}<0.001$. S1PR1, sphingosine-1-phosphate receptor 1 ; AML, acute myeloid leukemia; siRNA, small interfering RNA; DCF 2,7-dichlorofluorescein.

phorylation of ERK, whereas kinase activation appeared to be marginally attenuated in the S1PR1-knockdown cells (Fig. 4B). These results indicated that S1PR1 had a positive effect in the activation of ERK. In addition, the effects of the expression of S1PR1 on the phosphorylation of MKK1, the upstream kinase of ERK, was also examined and a similar result was obtained, suggesting that the enhancement of ERK activation was due tothe positive regulation of MKK1 signaling by S1PR1. Considering the roles of ERK signaling in cell growth and survival, it was hypothesized that the promotion of cell proliferation observed results, at least in part, from the positive modulation of MKK1-ERK signaling by S1PR1. Taken together, S1PR1 likely suppresses JNK signaling, leading to the promotion of myeloid leukemia cell apoptosis, and enhances cell proliferation by activating ERK signaling.

In conclusion, S1PR1 not only suppressed JNK signaling and promoted myeloid leukemia cell (mitochondrion-associated) apoptosis, but also facilitated cell proliferation, likely by suppressing the generation or accelerating the elimination of ROS, and by activating ERK signaling.

\section{Discussion}

AML is a heterogeneous clonal malignancy. The disease is characterized as multiple genetic alterations in hematopoietic 
A

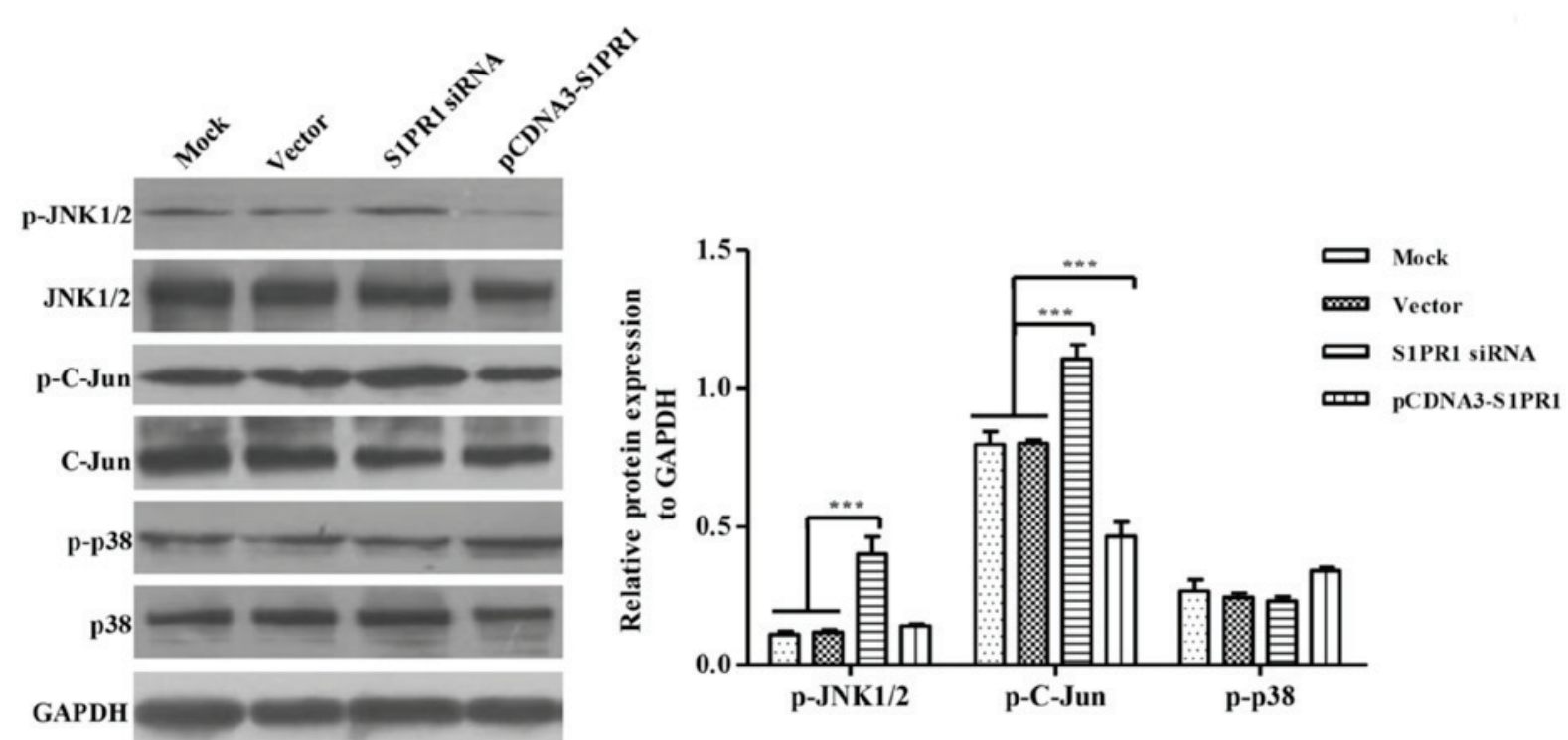

B

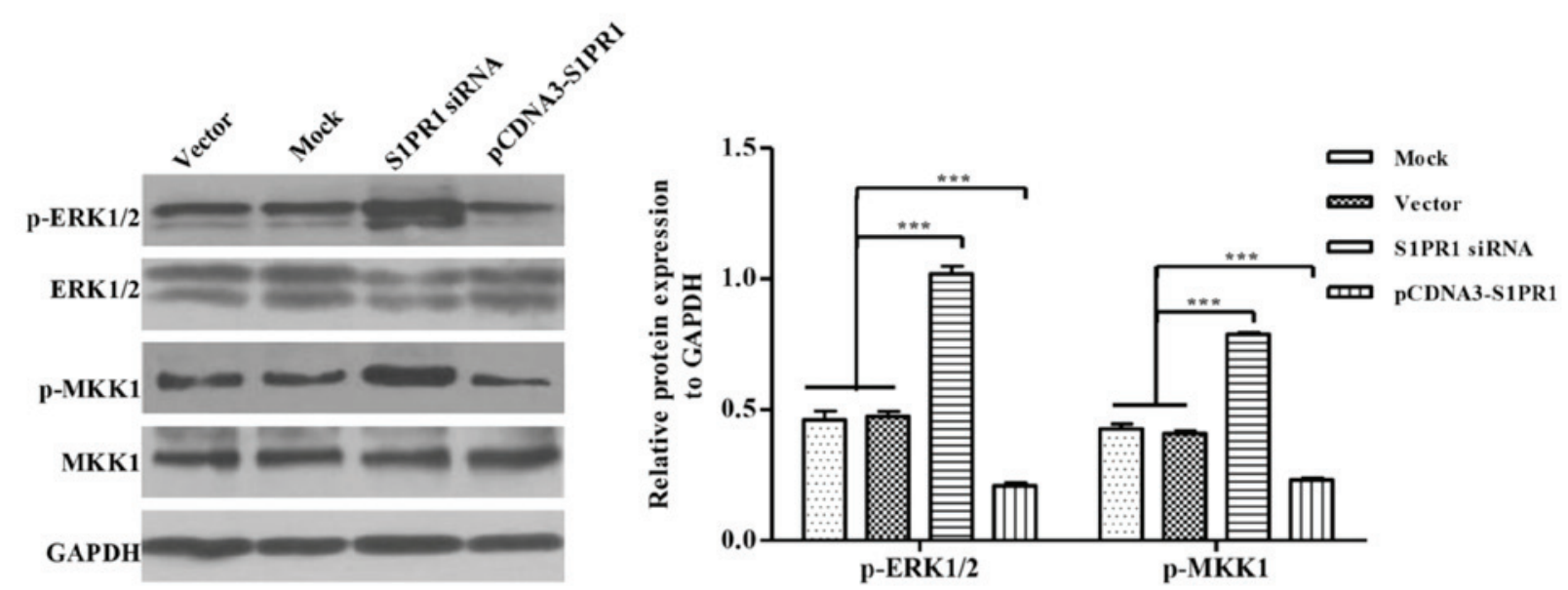

Figure 4. Western blot analysis. (A) S1PR1 specifically promoted the activation of JNK-c-Jun signaling. The cells were transfected with the indicated plasmids and lysed for western blot analysis at $24 \mathrm{~h}$ post-transfection using antibodies against the indicated proteins. GAPDH was detected as a control for sample loading. (B) S1PR1 stimulated MKK1-ERK signaling. Cells were transfected with the indicated plasmids and lysed for western blot analysis at $24 \mathrm{~h}$ post-transfection using antibodies against the indicated MKK1-ERK1/2 signaling proteins. GAPDH was detected as the control of sample loading. *** $<0.001$. S1PR1, sphingosine-1-phosphate receptor 1; JNK, c-Jun N-terminal kinase; MKK1; mitogen-activated protein kinase kinase 1; ERK, extracellular signal-regulated kinase; p-, phosphorylated; siRNA, small interfering RNA.

stem cells, including the translocation of a $\mathrm{t}(12 ; 18)(\mathrm{p} 13 ; \mathrm{q} 12)$ involving Ets Variant 6, which results in the overexpression of SET binding protein 1 and promotes the proliferation of leukemic cells (22). In previous years, several genetic markers in AML have been identified, leading to increased understanding and providing targets for treatment of the disease (23-26). However, the relevant aberrations that contribute to the pathogenesis of this disease remain to be fully elucidated. In the present study, the importance of S1PR1 in regulating the apoptosis and proliferation of AML cells were identified. S1PR1 inhibited AML cell apoptosis through interfering with mitochondrion-mediated apoptosis processes, ROS generation and JNK MAPK signaling. S1PR1 also promoted the proliferation of AML cells, which was at least partially due to the activation of ERK signaling by S1PR1.

ROS are diffusible chemical mediators. They can act directly on the apoptotic machinery, by accelerating depolarization and causing dysfunction of mitochondria (20), which in turn, function as a major source of ROS in the course of apoptosis (21). The results of the present study suggested that S1PR1 prevented mitochondrion-associated apoptosis and ROS generation, and confirmed the importance of S1PR1 as an anti-apoptotic factor, with elucidation of the corresponding mechanism. However, the upstream reason for the suppression of apoptosis remains to be elucidated, in terms of the reciprocal causation between mitochodria injury and ROS generation.

Impaired apoptosis can promote cancer cell survival. In the evolutionarily conserved 'stress' pathway-mitochondria signal transduction of apoptosis, the Bcl-2 family are core cell death machinery, and consist of pro-apoptotic proteins, including Bcl-2-associated death promoter and Bax, and anti-apoptotic proteins, including Bcl-2 and Bcl-extra large, which interact to maintain a balance (27). When anti-apoptotic Bcl-2 family members are overexpressed, the ratio of pro- and anti-apoptotic Bcl-2 family members is disturbed, which in turn potentially 
drives malignant progression and confers chemoresistance to cancer cells (23). According to the observations in the present study, S1PR1 inhibited mitochondrion-associated apoptosis by reducing the levels of Bax and preventing the cleavage of caspase-3 (Fig. 4). Although the inhibition of ROS generation by S1PR1 may explain the anti-apoptotic mechanism of the protein, there may be other factors involved in the S1PR1 knockdown-induced apoptosis of AML cells in addition to ROS.

MAPK signaling pathways are crucial in cell proliferation and apoptosis. Until now, three MAPK families have been clearly characterized: ERK1/2, JNK/MAPK and p38 kinase. The distinct MAPK members mediate different physiological activities; the ERKs function in proliferation and differentiation, whereas the JNKs and p38 MAPKs are predominantly involved in the stress response and apoptosis through responding to various stresses (25). Several studies have suggested that stimulated ERK1 activity results in enhanced cell proliferation $(24,26)$. Of note, S1PR1 can promote the MKK1/ERK signaling pathway, which was observed in the AML cells examined in the present study. Therefore, the enhancement of ERK activation may be a reason for the S1PR1-mediated promotion of AML cell proliferation observed in the present study. However, the mechanism by which S1PR1 enhances MKK/ERK signaling remains to be elucidated.

According to the results of the present study, the reduction of ROS is likely to be involved in S1PR1-induced cell proliferation. Although the S1PR1-induced reduction of ROS levels may be one reason for the inhibition of JNK signaling by S1PR1, the possibility that S1PR1 counteracts JNK activation in a ROS-independent manner cannot be excluded. Further investigations are required to determine whether S1PR1 suppresses JNK signaling by other mechanisms.

In conclusion, the present study identified the anti-apoptotic/pro-proliferative roles of S1PR1 in AML cells, and elucidated the corresponding mechanism. These findings may provide insights into the molecular mechanism underlying the progression and pathogenesis of AML, and benefit the development of therapeutical approaches for the disease.

\section{Acknowledgements}

This study was supported by the National Natural Science Foundation of China (grant nos. 81100362 and 81570148).

\section{References}

1. Pyne NJ and Pyne S: Sphingosine 1-phosphate and cancer. Nat Rev Cancer 10: 489-503, 2010.

2. Lee H, Deng J, Kujawski M, Yang C, Liu Y, Herrmann A, Kortylewski M, Horne D, Somlo G, Forman S, et al: STAT3-induced S1PR1 expression is crucial for persistent STAT3 activation in tumors. Nat Med 16: 1421-1428, 2010.

3. Chae SS, Paik JH, Furneaux H and Hla T: Requirement for sphingosine 1-phosphate receptor-1 in tumor angiogenesis demonstrated by in vivo RNA interference. J Clin Invest 114: 1082-1089, 2004.

4. Deng J, Liu Y, Lee H, Herrmann A, Zhang W, Zhang C, Shen S, Priceman SJ, Kujawski M, Pal SK, et al: S1PR1-STAT3 signaling is crucial for myeloid cell colonization at future metastatic sites. Cancer Cell 21: 642-654, 2012.
5. Yoshida Y, Nakada M, Harada T, Tanaka S, Furuta T, Hayashi Y, Kita D, Uchiyama N, Hayashi Y and Hamada J: The expression level of sphingosine-1-phosphate receptor type 1 is related to MIB-1 labeling index and predicts survival of glioblastoma patients. J Neurooncol 98: 41-47, 2010.

6. Matsuoka Y, Nagahara Y, Ikekita M and Shinomiya T: A novel immunosuppressive agent FTY720 induced Akt dephosphorylation in leukemia cells. Br J Pharmacol 138: 1303-1312, 2003.

7. Wang JD, Takahara S, Nonomura N, Ichimaru N, Toki K, Azuma H, Matsumiya K, Okuyama A and Suzuki S: Early induction of apoptosis in androgen-independent prostate cancer cell line by FTY720 requires caspase-3 activation. Prostate 40: 50-55, 1999.

8. Hung JH, Lu YS, Wang YC, Ma YH, Wang DS, Kulp SK, Muthusamy N, Byrd JC, Cheng AL and Chen CS: FTY720 induces apoptosis in hepatocellular carcinoma cells through activation of protein kinase C delta signaling. Cancer Res 68: 1204-1212, 2008.

9. Adams JM: Ways of dying: Multiple pathways to apoptosis. Genes Dev 17: 2481-2495, 2003.

10. Fiers W, Beyaert R, Declercq W and Vandenabeele P: More than one way to die: Apoptosis, necrosis and reactive oxygen damage. Oncogene 18: 7719-7730, 1999.

11. Simon HU, Haj-Yehia A and Levi-Schaffer F: Role of reactive oxygen species (ROS) in apoptosis induction. Apoptosis 5: 415-418, 2000.

12. Fiers W, Beyaert R, Declercq W and Vandenabeele P: More than one way to die: Apoptosis, necrosis and reactive oxygen damage. Oncogene 18: 7719-7730, 1999.

13. Chen YR, Shrivastava A and Tan TH: Down-regulation of the c-Jun N-terminal kinase (JNK) phosphatase M3/6 and activation of JNK by hydrogen peroxide and pyrrolidine dithiocarbamate. Oncogene 20: 367-374, 2001.

14. Sakon S, Xue X, Takekawa M, Sasazuki T, Okazaki T, Kojima Y, Piao JH, Yagita H, Okumura K, Doi T and Nakano H: NF-kappaB inhibits TNF-induced accumulation of ROS that mediate prolonged MAPK activation and necrotic cell death. EMBO J 22: 3898-3909, 2003.

15. Kamata H, Honda S, Maeda S, Chang L, Hirata H and Karin M: Reactive oxygen species promote TNFalpha-induced death and sustained JNK activation by inhibiting MAP kinase phosphatases. Cell 120: 649-661, 2005.

16. Grigoryev S, Salzberg A, Berg A, Harris-Becker A, Loughran T and Claxton D: Genome-wide mapping of large organized heterochromatin domains reveals hotspots of epigenetic and transcriptional changes associated with myeloid differentiation and acute myeloid leukemia (565.1). The FASEB Journal 28: 565.561, 2014.

17. Sarkisyan G, Gay LJ, Nguyen N, Felding BH and Rosen H: Host endothelial S1PR1 regulation of vascular permeability modulates tumor growth. Am J Physiol Cell Physiol 307: C14-C24, 2014.

18. Hamidi S, Schäfer-Korting $M$ and Weindl G: TLR2/1 and sphingosine 1-phosphate modulate inflammation, myofibroblast differentiation and cell migration in fibroblasts. Biochim Biophys Acta 1841: 484-494, 2014.

19. Wang T, Chen F, Chen Z, Wu YF, Xu XL, Zheng S and He X: Honokiol induces apoptosis through p53-independent pathway in human colorectal cell line RKO. World J Gastroenterol 10: 2205-2208, 2004

20. Jabs T: Reactive oxygen intermediates as mediators of programmed cell death in plants and animals. Biochem Pharmacol 57: 231-245, 1999.

21. Cai J and Jones DP: Mitochondrial redox signaling during apoptosis. J Bioenerg Biomembr 31: 327-334, 1999.

22. Cristóbal I, Blanco FJ, Garcia-Orti L, Marcotegui N, Vicente C, Rifon J, Novo FJ, Bandres E, Calasanz MJ, Bernabeu C and Odero MD: SETBP1 overexpression is a novel leukemogenic mechanism that predicts adverse outcome in elderly patients with acute myeloid leukemia. Blood 115: 615-625, 2010.

23. Adams J and Cory S: The Bcl-2 apoptotic switch in cancer development and therapy. Oncogene 26: 1324-1337, 2007.

24. Pagès G, Lenormand P, L'Allemain G, Chambard JC, Meloche S and Pouysségur J: Mitogen-activated protein kinases p42mapk and p44mapk are required for fibroblast proliferation. Proc Natl Acad Sci USA 90: 8319-8323, 1993.

25. Rubinfeld $H$ and Seger R: The ERK cascade: A prototype of MAPK signaling. Mol Biotechnol 31: 151-174, 2005.

26. Seger R and Krebs EG: The MAPK signaling cascade. FASEB J 9: 726-735, 1995.

27. Chalah A and Khosravi-Far R: The mitochondrial death pathway. Adv Exp Med Biol 615: 25-45, 2008. 